\title{
Téoros
}

Revue de recherche en tourisme

\section{Évolution et tendances des clientèles des bases de plein air}

\section{Jean Stafford}

Volume 8, numéro 2, juillet 1989

Hébergement et tourisme

URI : https://id.erudit.org/iderudit/1080323ar

DOI : https://doi.org/10.7202/1080323ar

Aller au sommaire du numéro

Éditeur(s)

Université du Québec à Montréal

ISSN

0712-8657 (imprimé)

1923-2705 (numérique)

Découvrir la revue

Citer cet article

Stafford, J. (1989). Évolution et tendances des clientèles des bases de plein air. Téoros, 8(2), 22-25. https://doi.org/10.7202/1080323ar d'utilisation que vous pouvez consulter en ligne.

https://apropos.erudit.org/fr/usagers/politique-dutilisation/ 


\section{Évolution et tendances des clientèles des bases de plein air}

Dans cet article, nous allons étudier l'évolution et indiquer les principales tendances de la clientèle des bases de plein air du Quebectl). Il s'agi de comparer deux enquêtes effectuées à des périodes différentes; la première a ćté réalisée en $1982^{212}$ et la deuxiéme en $1987^{15}$. Nous adoptons ici l'approche utilise par 0 . Duncan et $J$ Davis $^{[4]}$ dans les années $1975^{(5)}$.

Ceite approche consiste à refaire des enquettes en conservant les me̊mes critères méthodologi= ques (mèmes notionset definitions, Echantillons semblables, questionnaires identiques, etc.) de lâ première enquête. Les questions du questionnaire de recherche deviennent des indicateurs chronologiques; leurs variations sont, a ce moment, une mesure du changement dans ce secteur de la société.

\section{Méthodologie des enquêtes}

Pour les deux enquêtes, lobjectif general éait le même: cerner le profil socio-bconomique des usagers des bases de plein air, decouvrir leurs intérêts et les motiwations générales à utiliser ce type d'hébergement.

Les questionnaires, employés dans les deux recherches, comprenaient quatre dimensions importantes:

1- Ies variables socio-économiques usuelles (âge, sexe, scolarité, etc.), servant à la segmentation de la clientêle;

2. des variables mesurant les motivations des usagers à séjourner dans une base de plein air:

3- une troisiẻme dimension axếe sur l'évaluation de la satisfaction des clients par rapport aux services offerts par les bases:

4- enfin, une quatrième dimension servait à identifier les sources d'informations des usagers (télevision, radio, journaux, revues).

Aussi, le deuxièmé questionnaire comprenait près de $85 \%$ des questions ayant déjà servies. dans le premier questionnaire.

Les deux questionnaires ont eté administres, pour entrevues téléphoniques, par l'équipe de recherche. Aussi, dans les deux cas, il s'agit d'echantillons aléatoires. Les bases de sondages des deux enquêtes ont été établies à partir des fiches d'inscriptions de l'année avant l'enquếte; par exemple, pour la deuxieme recherche, l'année de référence des fiches consuliées est 1986.
A partir des fiches d'inscription, la population échantillonnée a éte tirée au hasard selon un intervalle d'ćchantillonnage determiné par le nombre de fiches disponibles.

La taille de l'echantillon de 1982 était de 647 repondants et celle de 1987 de 889 répondants. Dans le premier cas, le taux de réponse a été de $79,8 \%$ et dans le second cas de $73,3 \%$. Dans les deux études, la marge d'ereur thérique sur les réponses était de $4 \%$ à un seuil de confiance de $95 \%$.

\section{Évolution des clientèles de 1982 à 1987}

Nous allons analyser l'évolution des clientẻles des bases de plein air pour les perriodes 1982 1987 à l"aide de certaines variables factuelles telles que l'âge, la scolarité, le secteur de travaill, l'śtat civil et la région de provenance des usagers.

Dans toutes les recherches sur l'hébergement, l'äge est une variable essentielle; dans le tableau 1 , nous pouvons mesurer l'bolution accomplie dans les strates d'âge durant ces périodes.

On remarque ici la saignée du groupe d'âge des 24 ans et moins qui disparaît presque complètement: it s'agissalit auparavant de près du tiers de laclientèle. Ce bouleversenent est dû probablement à la hausse des prix qui a fait fuir les étudiants et les jeunes moins fortunés. Le groupe des $25-29$ ans diminue aussi de façon sensible. I] y a aussi une brusque flambée des 41 ans el plus qui passent de $8,5 \%$ à $24,2 \%$ de l'ensemble des usagers.

Dans le tableau 2, nous présentons l'évolution des niveaux de scolarite entre les deux périodes choisies.

Ici, les changenents sont moins importants entre 1982 et 1987: il y a une atugmentation lente de la scolarisation générale de la clientèle. En 1987. les diplômés universitaires regroupent $51,4 \%$ de la clientèle des bases de plein air.

Le secteur de travail (tableau 3 ) des répondants montre lui aussi des changements marquês.

Lesecteur public augmenteen passant de $18,3 \%$ de l'ensemble à $45,4 \%$. Le secteur privé est amputé de près de $50 \%$ de ses effectifs $(4 \mathrm{I} \%$ en 1982 et $19,1 \%$ en 1987). En 1987, le secteur public et le secteur para-public forment $80_{4} 9 \%$ 
de la clientele.

Dans le tableau 4 , nous remarquons que le pourcentage des célibataires diminue entre les deux périodes étudiées.

Cette évolution correspond aussi à l'augmentation du nombre des enfants chez les répondants et a la diminution importante des moins de 30 ans.

On assiste aussi aै des transformations dans la provenance géographique des répondants entre 1982 et 1987 (tableau 5).

La région de Montréal accapare une partie de plus en plus grande de la clientẻle des bases de plein air. La part de la région de Québec augmente elle aussi trés légerement. Les autres régions du Québec sont les grandes perdantes de cette évolution.

\section{La motivation et la satisfaction}

Afin d'étudier, plus en profondeur, la motivation et la satisfaction des usagers des bases de plein air, nous avons utilisé l'analyse factorielle. Dans l'analyse factoriellen "Le but premier est de trouver un moyen de condenser l'information contenue dans le nombre et de variables d'origine, dans un ensemble plus petit de nouvelles variables en perdant le minimum d'informations" ${ }^{\text {"th }}$, Cette technique permet aussi d'évaluer et de pondérer l'importance des sous-groupes explicatifs ainsi crés.

Lobjectif de l'analyse factorielle est d'analyser les relations statistiques observees entre un ensemble de variables en déterminant un petit groupe de "facteurs" explicatifs qui résument ces relations. Dans l'analyse factoriclle, on fait done l'hypothèse implicite que les relations entre plusieurs variables se référent à un élëment commun (le facteur) quil rend compte de ces affinites. Cette méthode nous amene aussi a définir, de façon hiérarchique, ces sous-groupes de variables (les facteurs) et démontrer leur valeur explicative ${ }^{[7]}$.

Dans le tableau 6, nous anvons l'analyse factorielle des raisons qui motivent le choix d'une base de plein air. Le facteur I regroupe les variables reliées aux activités de plein air; ce premier facteur "explique" $50,3 \%$ de l'ensemble des motivations a choisir une base de plein air. Le facteur II: les services offerts et les prix representent pres de $18 \%$ des motivations totales.

Le type de vacances est le thème du troisième facteur: les vacances familiales ont le haut du pavé avec .88. Les vacances seul (ou seule) n'a qu'un coefficient (négatif) de -22; ce troisieme facteur compte pour $13,4 \%$ des motivations globales a choisir une base de plein air.

TABLEAU 1

\section{L’âge des répondants en 1982 et en 1987}

\begin{tabular}{|c|c|c|}
\hline Années & 1982 & 1987 \\
\hline 24 ans ef moins & 23,8 & 2,9 \\
\hline $25-29$ ans & 27,0 & 18,2 \\
\hline $30-40$ ans & 40,7 & 54,7 \\
\hline 41 ans et plus & 8,5 & 24,2 \\
\hline TOTAL: & 100 & 100 \\
\hline
\end{tabular}

TABLEAU 2

La scolarité des répondants en 1982 et 1987

\begin{tabular}{|l|c|c|}
\hline Secolarite Annés & 1982 & $\mathbf{1 9 8 7}$ \\
\hline Secondaire et moins & 24,8 & 21,8 \\
\hline Collégial & 32,8 & 26,8 \\
\hline Premier cycle universilaire & 33,7 & 38,6 \\
\hline Deuxieme cycle universitaire & 8,7 & 12,8 \\
\hline TOTAL: & 100 & 100 \\
\hline
\end{tabular}

TABLEAU 3

Le secteur de travail des répondants en 1982 et en 1987

\begin{tabular}{|l|c|c|}
\hline Secteur & 1982 & 1987 \\
\hline & & \\
\hline Public & 18,3 & 45,4 \\
\hline Pura-public & 40,7 & 35,5 \\
\hline Privé & 41,0 & 19,1 \\
\hline TOTAL: & 100 & 100 \\
\hline
\end{tabular}

TABLEAU 4

\section{L'état civil des répondants en 1982 et en 1987}

\begin{tabular}{|l|c|c|}
\hline État civil Années & 1982 & 1987 \\
\hline Célibataire (seul) & 43,0 & 30,8 \\
\hline Marié ou en union libre & 57,0 & 69,2 \\
\hline TOTALi & 100 & 100 \\
\hline
\end{tabular}

TABLEAU 5

La provenance géographique des répondants en 1982 et en 1987

\begin{tabular}{|c|c|c|c|}
\hline Régions & Annees & 1982 & 1987 \\
\hline Montreal & & 56,4 & 62.6 \\
\hline Québec & & 18,2 & 20.7 \\
\hline Autres & & 25,4 & 16.7 \\
\hline TOTAL: & & 100 & 100 \\
\hline
\end{tabular}


TABLEAU 6

\section{Analyse factorielle des raisons qui motivent le choix d'une base de plein air (matrice factorielle après rotation varimax)}

\begin{tabular}{|c|c|c|c|}
\hline Facteur I & & Activites de plein air & \\
\hline Initiation au plein air & .67 & Valeur propre & 2.58 \\
\hline Acoessibilite aux equipements & 54 & F de la variance & 50.3 \\
\hline La variété des activités & .52 & & \\
\hline Nouvelle forme de vacances & .35 & & \\
\hline Facteur II & & Rapport services/prix & \\
\hline Services offerts & .56 & Valeur propre & .92 \\
\hline Prix du séjour & .45 & w de la variance & 17.9 \\
\hline Facteur III & & Vacances & \\
\hline Vacances familiales & .88 & Valeur propre & .69 \\
\hline Vacinces seul & -22 & $\%$ de la variance & 13.4 \\
\hline Facteur IV & & Dimensions extérieures & \\
\hline Rencontrer des gens & .52 & Valeur propre & 55 \\
\hline Animation & .40 & \% de la variance & 10.5 \\
\hline Se perfectionnier (plein air) & .36 & & \\
\hline Attrait de la region & .35 & & \\
\hline Facteur $\mathrm{V}$ & & Dimensions intérieures & \\
\hline Repos & .61 & Valeur propte & .41 \\
\hline Acces à la nature & .33 & o de la variance & 7.9 \\
\hline Vacances en couple & .25 & & \\
\hline
\end{tabular}

TABLEAU 7

Analyse factorielle de la satisfaction (matrice factorielle après rotation varimax)

\begin{tabular}{|c|c|c|c|}
\hline Facteur I & & Label plein air & \\
\hline Animation des soires & .77 & Valeur propre & 6.37 \\
\hline $\begin{array}{l}\text { Animation des activites } \\
\text { de plein air }\end{array}$ & .75 & \% de la variance & 64.7 \\
\hline $\begin{array}{l}\text { Competence des } \\
\text { animateurs }\end{array}$ & .73 & & \\
\hline Activités sociales & 69 & & \\
\hline Nombre des animateurs & .62 & & \\
\hline Activités de plein air & .61 & & \\
\hline Ambiance générale & .49 & & \\
\hline Sécuritế & 38 & & \\
\hline Prix du séjour & .29 & & \\
\hline Facteur II & & Restauration & \\
\hline Qualité de lalimentation & .84 & Valeur propre & 1.83 \\
\hline Equilibre des menus & .79 & $\%$ de la variance & 18.5 \\
\hline $\begin{array}{l}\text { Quantite des portions } \\
\text { alimentaires }\end{array}$ & .67 & & \\
\hline Facteur III & & Hebergement & \\
\hline Confort & .88 & Vuleur propre & 1.17 \\
\hline Type d'hébergement & .85 & \% de la variance & 12.6 \\
\hline Propreté (hébergement) & .63 & & \\
\hline Facteur IV & & Facteur résiduel & \\
\hline Propreté (salle à manger) & .58 & Valeur propre & .46 \\
\hline $\begin{array}{l}\text { Ambiance (salle à } \\
\text { manger) }\end{array}$ & .44 & of de la variance & 4.2 \\
\hline Altrail du site & 32 & & \\
\hline Indications routiènes & .26 & & \\
\hline
\end{tabular}

Les dimensions extérieures correspondent à $10,5 \%$ de l'explication totale et les dimensions interieures $7,9 \%$. Les deux premiers facteurs sont vraiment les plus importants car, it eux deux, ils resument $68,2 \%$ de l'ensemble des motivations à visiter les bases de plein air. En fonction de ces résultats, on peut facilement penser que les autres facteurs sont secondaires dans l'analyse générale desmotivations; les autres facteurs (III, IV et V) peuvent quand meme avoir une plus grande valeur au plan local.

Si l'on compare ces résultats à ceux de 1982, on retrowwe de légères variations; par exemple; l'initiation aux activites étaient dans le facteur I en 1982, le prix par contre se trouvait dans le facteur I et est actucllement dans le facteur II. II faut aussi souligner que les vacances familiales se trouvaient dans le facteur $V$ en 1982 (dans le troisiene aujourd'hui avee $13,4 \%$ de la variance tolale); il s'agit donc dans ce cas d'une remontéc spectaculaire.

A lepoque aussi, les deux preniers facteurs representaient $78 \%$ de la variance totale (pour $64 \%$ aujourd hui). II y a done de lents changementsentre 1982 (année de la première enquête) et 1987; on peut constater un certain étalement des motivations à choisir une base de plein air comme sejour de vacances.

Le tableau 7 contient les résultats de l'analyse factorielle de la satisfactiondes usagers en fonction de leur demier séjour dans une base de plein air, Le premier facteur que nous appelons "label plein air" résume toutes les variables qui expriment le mieux ce que sont les bases de plein air, c'est-à-dire les activites de plein air, l'animation dans un cadre sécuritaire avec un prix raisonnable. On voit que ce facteur "explique" $64,7 \%$ de la variance totale.

Ce mềme facteur, dans l'étude de 1982, ne représentait que $46 \%$ de l'ensemble de la variance: il y a done la un bond important dont il faudrait tenir compte! On peut remarquer aussi que l'ambiance générale dans les bases et le prix se retrouvaient dans le sondage de 1982, dans le facteur VI ef ne comptait (avec d'autres variables) que pour $4,5 \%$ de la variance totale.

La restauration (les divers aspects de lalimentation) forme le deuxième facteur; il "explique" $18,5 \%$ de lensemble de la satisfaction. En 1982. ce pourcentage était de $18,1 \%$, il n'y a done pas ici de variation. Les deux premiers facteurs réunis monopolisent donc $83,2 \%$ de la satisfaction totale $(64.1 \%$ pour les deux en 1982). Les autres facteurs ont donc beauocup moins d'impact sur la satisfaction qu'en 1982.

Le troisieme facteur, lhébergement, represente $12,6 \%$ de la satisfaction totalc; l'hébergement formait le cinquième facteur en 1982 et "expliquerait" $7,3 \%$ du total de la satisfaction à séjourner dans une bage de plein air. 
Enfin, le facteur résiduel, le quatrième, est un sous-groupe fourre-tout quil conprend des variables assez hétéroclites. L'ensemble de ce facteur ne compte que pour $4,2 \%$ de la satisfaction. II faut indiquer, en teminant, qu'en 1982. cette analyse comptait sept facteurs alors que celle de 1987 n'en compte que quatre. Ce tassement du nombre des facteurs limite l'explication à trois facteurs: le label de plein air, la restauration et l'hébergement; les elements résiduels foumissent très peu d'information sur la satisfaction des usagers.

\section{Les tendances de la clientèle aujourd'hui}

A la lumiere de ces comparaisons entre 1982 et 1987, on peut tracer certaines tendances de la clientèle des bases de plein air:

- les usagers sont de moins en moins jeunes; les $30-40$ ans dominent comme ils dominent la pyramide des âges de la population globale. Le vieillissement de la population générale du Québec a done des effets directs sur la clientele des bases de plein air. On peut se demander, si a long terme, les bases de plein air peuvent survivre à cette saignée des jeunes ( 24 ans et moins) et des moins jeunes (25 a 29 ans)? La perméabilité de cette clientèle au vieillissement généralisé dé la population québécoise est assez inquiétante pour l'avenir.

- la scolarisation de la clientele est de plus en plus intensive et se démarque pas mal de l'ensemble de la population québecoise.

le secteur public et le secteur para-public monopolisent près de $80 \%$ de la elientèle; c'est une forte évolution depuis 1982. Le secteur privé est réduit à la portion congrue; si cette tendance se maintenait, la clientèle provenant de cesecteur pourrait disparaitre à moyen terme.

Enfin, l'origine de la clientèle vient de plus en plus de milieux urbains.

\section{Les conséquences de ces ten- dances}

Les wsagers des bases de plein air sont des hommes et des femmes entre 30-40 ans, tres fortement scolarisés, venant des milieux urbains. Ces usagers font partic des classes moyennes supérieures et des classes supérieures. Cette clientele correspond à une géneration issue du "baby boom", orientée vers l"ecologic, la nature et le grand air. C'est une génération qui tient actuellement le haut du pavé, qui faitet défait les modes et exige ce qu'il y a de mieux.

L'initiation au plein air, l'accessibilité aux équipements et la variété des activités demeurent des éléments-clés de la motivation à séjourner dans une base de plein air. A ces éléments s:ajoutent les services offerts te le prix du séjour. Ce sont les variables lourdes qui orientent et maintiennent les choix de séjour et assurent la fidélisation de la clientèle.

La satisfaction des usagers repose aussi sur des valeurs sûrcs: l'animation, l'ambiance générale et le prix du séjour. L'allimentation est aussi une valeur forte; ces quelques variables sont les veritables pivots de la satisfaction. Ce sont des aspects traditionnels qui fonment l'image de marque des bases de plein air, ce sont elles qu'il fatut publiciser et pousser de l'avant.

L'étude des tendances de la clientèle entre 1982 et 1987 pose de serieuses questions pour le moyen terme et le long terme. Le vieillissement de la clientèle deviendra sous peu un problène lancinant. Une clientèle plus âgểe aura des exigences plus grandes au niveau du confort dans l'hébergement et de la qualité de l'alimentation. Nous savons aussi que ce sont les jeunes (moins de 30 ans) qui sont les plus nombreux chez les fanatiques et les mordus des sports de plein air; ce sont eux qui restent le plus longtemps dans les bases (nuitées) et qui viennent le plus souvent. II faudrait penser à des programmes speciaus pour attirer les jeunes entre 18 et 30 ans.

L'augmentation de la scolarisation et l'acces aux professions supérieures par les usagers des bases de plein air peut aussi poser des problemes inédits. Les répondants trés scolarises ont l'esprit plus critique que les autres groupes et sont moins entichés des sports de plein air traditionnels.

Le monopole des secteurs publics et para-publics peut être un autre sujet d'inquiétude bien qu'il soit difficile a śvaluer à court terme. La perte de vitesse de ces secteurs en termes du nombre des emplois et du prestige social (dans la société québécoise) peut avoir des effets indirects autant sur les nuitées que sur le nombre de sejour.

La concentration des clientèles dans les villesde Montréal et de Québec est-elle un bon signe? Il est difficile de se prononcer mais on peut penser qu'il y aurait un certain intérêt à diversifier la clientèle dans sa composition géographique. Cette reflexion s'applique aussi à la stratification par åge, à la scolarité et aux strates socioprofessionnelles.

Les bases de plein air ont des points forts et des caractéristiques particulières; ces éléments font la force de l'image de marque des bases de plein air. Cette marque de commerce occupe une place originale dans lindustrie du tourisme. II est important de renforcer certains de ces blements et de les mettre plus en valeur.

II sembleque certaines caractéristiques typiques de la clientèle des bases de plein air tendent, avec le temps, à s'exacerber. Il devient urgent de diversifier cette clientẻle et de faire profiter d'autres groupes sociaux de ce concept touristique particulier.

\section{NOTES EXPLICATIVES}

(1) Nous parlons an fain des 13 bases de plein air affiliees a I'Associanion Reseau Plein Air.

(2) Voir: Safford, J., Prufil socio-economique des usagers des bases et des centres de plein air du Quebec. Deparentend d'ctudes urbaines el tourisiques, UOAM, Monutral, 1083.

(3) Voir: Stafford. J., Etude et aral hat socio-economique de la clientele des bases nembres de Reseau PleinAir. Detpartement d'tudes umbaines et iouristiques. UOAM, Montreal, $19 \mathrm{gg}$.

(4) Voir: Duncan, O. Measwiag Social Change wa Reph.

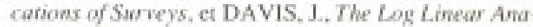
bysis of Surney Replicanoms, dans Secial Indicators Models, Ruskell Sage Foundations, N. Y, 1975.

(5) Murcel Samwon, en tourisme, ardalise une reprise d'etudesemblablece comagarant des rtsul ate de 1970 a ceus de 1980; wir: SAMSON.M. Nacances et tourisme 1980 Eraks et documente \#30. INRS-Utbanisation, Mor. intal, 1982.

(6) GREEN, P., FRANCE, R., Marketing et méthodes quantitatives. Dunxd, Paris, 1973,

(7) Voir KIM. J., MUELLER. C. Introduction to Factor Analysis, Sage Publicutions, Bewerly Hills, 1978. 